\title{
Irradiation of benzene molecules by ion-induced and light-induced intense fields
}

\author{
D. Mathur \\ Tata Institute of Fundamental Research, Homi Bhabha Road, Mumbai 400 005, India
}

(February 9, 2020)

\begin{abstract}
Benzene, with its sea of delocalized $\pi$-electrons in the valence orbitals, is identified as an example of a class of molecules that enable establishment of the correspondence between intense ion-induced and laser-light-induced fields in experiments that probe ionization dynamics in temporal regimes spanning the attosecond and picosecond ranges.
\end{abstract}


Matter is inherently unstable when exposed to electric fields whose magnitudes approximate interatomic Coulombic fields. Studies of the response of matter to very intense fluxes of such fields address fundamental issues concerning the physics of systems driven strongly away from equilibrium. There are two approaches that enable terrestrial access to such fields, and their use in probing the ionization (and dissociation) dynamics of atoms (and molecules): use of fast, highly-charged beams of heavy ions on the one hand, and focused, intense laser light on the other. The equivalence of the two approaches has only been explored in somewhat desultory fashion. The interaction of atoms and molecules with intense fields is clearly a complex nonperturbative, dynamical problem and prospects of rigorous theoretical treatment remain remote. Experimental information, albeit of a morphologial nature, is, therefore, of considerable importance and interest. Specifically, it is necessary to identify a class of target species whose properties make them suitable for studies aimed at disentangling the important time-dependent aspects of the field-target interaction from those that are 'static' or structure-dependent, so that the prospects of gaining qualitative insights are improved. The time-dependent aspect of the field-target interaction is especially of relevance in the case of molecules. Contemporary laser technology makes available intense light fields in pulses of picosecond $(p s)$ and femtosecond $(f s)$ duration. For molecules, these time scales are of significance in relation to typical rotational and vibrational time periods (tens of $p s$ and tens of $f s$, respectively). In the case of ion-induced fields, however, a beam of highly-charged ions traverses a distance of a few $\AA$ in only a few tens of attoseconds as, thereby opening prospects of studies of molecular dynamics on times scales in which even vibrational motions can be regarded as "frozen". Moreover, these ultrashort times imply enormous uncertainties in energies, thereby opening new avenues of molecular dynamics studies that fall well outside the ambit of conventional molecular quantum mechanics. We report in this Letter results of a study that suggests that benzene, with its sea of delocalized $\pi$-electrons in the valence orbitals, constitutes a molecule that enables establishment of the correspondence between intense ion-induced and laser-light-induced fields in experimental probes of the ionization dynamics in temporal regimes that span the as and $p s$ ranges.

The correspondence between ion-induced and light-induced fields can most usefully be established by considering three temporal regimes. In the case of relativistic ion beams (whose energy $\sim \mathrm{GeV} /$ nucleon, $\beta=v / c \sim 1$ ) colliding with atoms or molecules, the WeizsäckerWilliams equivalent photon model [1] transforms the Coulombic field in the projectile's rest frame to the target's rest frame, and equates the effect of the field to two, orthogonallydirected photon pulses. Application of such equivalent-photon pulses to atomic ionization have recently attracted attention [2]. At the opposite temporal extreme, purely electrostatic considerations use Coulomb's law; for instance, in the case of $\mathrm{Si}^{3+}$ projectiles, a target atom experiences a field of magnitude $\sim 5 \mathrm{~V}^{-1}(\sim 0.1$ a.u. $)$ at a distance of $3 \AA$. In the intermediate regime, one that is experimentally most accessible using highly-charged ion beams of $\mathrm{MeV}$ energy, the effective ion-induced electric field experienced by a target may be deduced from the Poynting vector: $I_{e f f}(t)=\frac{1}{\mu_{0}}|\vec{E}(t) \times \vec{B}(t)|$. Following the pioneering work of Rhodes and coworkers in this regime [3], recent work has focused on observing similarities and differences between ion-induced and laser-induced ionization patterns in molecules like water and chloromethanes of different symmetries 迎. For $\mathrm{H}_{2} \mathrm{O}$, the morphology of the dissociative ionization pattern was found to be grossly different in the two cases. Differences in the case of the chloromethanes were much less, but were still significant. These differences 
may be rationalized either by noting that the directional properties of the applied field are different in the two cases, or by invoking the different temporal profiles of the applied fields. Although the magnitudes of the ion-induced and light-induced fields were almost identical in these experiments, the directional properties of the two types of field were different in the following sense: the laser light was linearly-polarized and so, the direction of the lightinduced field was constant in the course of the interaction, unlike the time-dependence that is intrinsic to the direction of the ion-induced field. There is some evidence that, in the case of laser-molecule interactions, the directional properties of the applied field can influence molecular dissociation pathways [5]. Other factors, such as indirect ionization events (involving intermediate electron capture and loss) in the case of the ion-impact experiments, can be discounted by judicious choice of collision energy (and, as indicated below, by supplementary measurements made, under identical operating conditions, on well-understood collision systems involving rare-gas targets).

Before presenting results that demonstrate the good correspondence between ion-induced and light-induced ionization patterns of $\mathrm{C}_{6} \mathrm{H}_{6}$, it is important to address other facets of the equivalence we explore, namely the peak field intensity and the temporal/spatial features for the two types of fields. Our ion-impact experiments used fast beams of mass-selected, highlycharged ions $\left(\mathrm{Si}^{q+}, q=3,8\right.$ at an energy of $\left.50-100 \mathrm{MeV}\right)$ that were obtained from a tandem accelerator. Slow recoil ions resulting from large impact-parameter interactions between the projectile ions and $\mathrm{C}_{6} \mathrm{H}_{6}$ vapor were extracted, with unit collection efficiency, into a linear time-of-flight (TOF) spectrometer located in a direction that was orthogonal to the incident ion beam. The methodology has been described previously [四]. Experiments were also performed with $\mathrm{F}^{7+}$ projectiles at an energy of $110 \mathrm{MeV}$, and in these the ion analysis and detection was with quadrupole mass filter (all other experimental features being similar). In the laser-based experiments, light pulses (of 35 ps and 100 fs duration), from high-intensity Nd:YAG and Ti:sapphire lasers, were focused by a biconvex lens of $10 \mathrm{~cm}$ focal length such that peak intensities within the focal volume were in the range $10^{12}-10^{16} \mathrm{~W} \mathrm{~cm}{ }^{-2}$. Ions produced in the focal volume were extracted, again at $90^{\circ}$ to the incident laser beam, and analyzed by a 2 -field TOF set-up.

One important facet of the comparison of ion-induced and light-induced ionization dynamics is the quantification of the peak magnitude of the applied field. A limiting value of the impact parameter, $b$, has to be deduced for each ion-collision system. However, for molecular targets, $b$ remains a somewhat elusive parameter. The range of $b$-values that come into play manifests itself in the mean recoil energy $\left(E_{r}\right)$ that is imparted to each molecular ion that is created in the interaction. This, in turn, is reflected in the temporal width of molecualar ion peaks in the measured TOF spectrum. In the case of $C_{6} H_{6}^{+}$ions formed in our experiments with $\mathrm{Si}^{q+}(q=3,8)$ projectiles, we measured $E_{r}$ to lie in the range $30-40 \mathrm{meV}$. There is an established method [6], based on classical trajectory Monte-Carlo techniques [7], that enables deductions to be made of the impact parameter dependence of multiple ionization probabilities in fast-ion collisions. A value of $3 \AA$ was deduced as the lower limit for $b$ in our experiments. Collisions that occur at smaller $b$-values give rise to recoil energies far in excess of $E_{r}$, and these are discriminated against by the angular resolution of the spectrometers used by us. We confirmed the veracity of our deductions of $b$ by determining total cross sections for formation of low-energy $\mathrm{Ar}^{q+}$ recoils $(q=1-10)$ in the same apparatus. Deduction of $b$ can also be complicated by electron capture and loss processes that might 
influence the formation of recoils in charge state $q>1$. However, it was confirmed that for a range of ions (namely, $\mathrm{Si}^{q+}, q=3-12$ ), direct ionization dominated the overall dynamics. By way of example, we cite the measured the total cross section for $\mathrm{Ar}^{4+}$ formation in $\mathrm{Si}^{10+}-\mathrm{Ar}$ collisions to be $8 \times 10^{-17} \mathrm{~cm}^{2}$; corresponding cross sections for $\mathrm{Ar}^{4+}$ formation accompanied by 1-electron capture and loss were $3 \times 10^{-18} \mathrm{~cm}^{2}$ and $7 \times 10^{-19} \mathrm{~cm}^{2}$, respectively. For $C_{6} H_{6}{ }^{-}$ ion collisions at $50-110 \mathrm{MeV}$, the domination of direct ionization processes is likely to be even more pronounced.

For the light-field experiments, determination of the peak field value is somewhat less difficult, but note the following facet that has hitherto not been widely acknowledged. Just as the ion- $C_{6} H_{6}$ interaction accesses a range of $b$-values $(b>3 \AA)$, and, hence, exposes the molecules in the interaction zone to a corresponding range of applied fields, so in the laser case there is a spatial distribution of intensities that gives rise to a corresponding distribution of fields. Such spatial distribution of the laser-induced field, and the ion distribution that results from it, was measured using a method that has recently been described in detail [8]. The spatial field distributions obtained in both the ion-impact and laser-based experiments are shown in Figs. 1 and 2, respectively. For convenience of comparison, we express the field magnitude obtained in the ion-impact experiments in terms of an effective intensity that can be directly compared with an easily-determined parameter in the laser experiments, namely the light intensity. Judicious choice of operating conditions enable the spatial field distributions to be very similar in the two sets of experiments. The examples shown are for a $100-\mathrm{MeV}$ beam of $\mathrm{Si}^{8+}$ ions, readily accessed in tandem accelerators, and for a $100-$ fs long laser pulse of peak intensity $2 \times 10^{15} \mathrm{~W} \mathrm{~cm}^{-2}$, accessed by focusing a $1 \mathrm{~mJ}$ beam of $806 \mathrm{~nm}$ wavelength from a Ti:sapphire laser to a spot size of $\sim 25 \mu \mathrm{m}$.

Fig. 3 shows typical ionic fragmentation patterns obtained when $C_{6} H_{6}$ is irradiated by $100-\mathrm{MeV} \mathrm{Si}^{3+}$ ions and 35-ps-long laser pulses of $532 \mathrm{~nm}$ wavelength. The peak intensity in the latter case was $\sim 8 \times 10^{13} \mathrm{~W} \mathrm{~cm}^{-2}$ and, by using a small $(2 \mathrm{~mm})$ aperture at the entrance of our TOF spectrometer, the lower part of the intensity range that we accessed was $\sim 5 \times 10^{12} \mathrm{~W} \mathrm{~cm}^{-2}$. In the case of the ion impact data, the peak value of effective intensity at an impact parameter of $3 \AA$ was $\sim 5 \times 10^{14} \mathrm{~W} \mathrm{~cm}^{-2}$. We also conducted experiments with $\mathrm{Si}^{8+}$ and $\mathrm{F}^{7+}$ ions, and with 100-fs duration laser pulses (of $806 \mathrm{~nm}$ wavelength), with peak intensities in the range $10^{13}-5 \times 10^{16} \mathrm{~W} \mathrm{~cm}^{-2}$. The gross features of the measured ionic fragmentation patterns remained essentially unaltered under all these conditions, although the highest laser intensities gave rise to a larger degree of multiple ionization (that could also be directly correlated with ion beam data). Illustrative data are shown in Fig. 4, for 110 $\mathrm{MeV} \mathrm{Si}{ }^{8+}$ impact and irradiation by $100 \mathrm{fs}$ laser pulses of intensity $5 \times 10^{15} \mathrm{~W} \mathrm{~cm}^{-2}$. Here, a larger $(15 \mathrm{~mm})$ aperture was used at the entrance of our TOF spectrometer in our laser experiments, thereby giving access to the entire intensity range shown in Fig. 2. Recent work [8] has shown that the lower-intensity portions of this range dominate the overall dynamics because a very much larger proportion of the focal volume that is accessed by the spectrometer samples the lowest intensities. Under these circumstances, the contribution of the parent $\mathrm{C}_{6} \mathrm{H}_{6}{ }^{+}$ion to the mas spectrum is very much enhanced (not shown in the figure). The central portion of the focal volume samples the peak intensity region and, consequently, multiple ionization events are also more in evidence in the spectrum shown in Fig. 4. A noteworthy feature is the somewhat unexpected preponderence of $\mathrm{H}^{+}$and $\mathrm{H}_{2}^{+}$fragments, indicating that extremely energetic proceeses occur in this intensity regime that overcomes 
the intrinsic strength of the aromatic ring structure and causes extensive fragmentation, opening dissociation channels that are seldom accessed in electron impact experiments. In order to preclude the possiblity of diverting from the main focus of attention in this Letter, we refrain from discussing the details of the ionic fragmentation patterns (such discussion can be found in the context of $n s$ and $p s$ laser-induced fragmentation of $C_{6} H_{6}$ in ref. [9]). We focus on the fact that the data presented here clearly does show a surprising degree of similarlity in the morphology of the ion-induced and light-induced fragmentation patterns.

So, what lessons are to be drawn from our attempts to study the correspondence between ion-induced and light-induced strong fields and their effect on molecular dynamics? Clearly, the first lesson is that the observed similarities in the mass spectra show that this correspondence is worthy of further pursuit, notwithstanding the "obvious" differences in the nature of the fields generated by non-relativistic charged particle beams and pure electromagnetic radiation. The second lesson originates in the practical consideration that irradiation of matter by either type of field entails exposure to a range of field intensities; single-valued fields are unlikely to be encountered, either in nature or in the laboratory. The spatial variation of field intensity gives rise to "focal volume effects" amd makes it mandatory to ensure that equivalent intensity ranges are accessed in studies of field-matter interactions in order to make meaningful comparisons about the effects on the overall dynamics of the time duration of each type of field. We believe that we have succeeded in achieving this equivalence in our experiments. The somewhat surprising result to emerge is that, at least for $C_{6} H_{6}$, whether the intense field is of picosecond or attosecond duration is of little consequence as far as the overall ionic fragmentation pattern is concerned. Thirdly, the other major difference between the two types of fields concerns their directional properties: the laser-induced field has a well-defined direction as it originate in light that is linearly-polarized. But, the direction of the ion-induced field changes in the course of the collision process. Our data indicate that these properties seem unimportant to the overall dynamics. It may be argued that for ion-induced fields the change of direction is too fast to be of consequence.

Can our results be interpreted within the framework of molecular quantum mechanics? One attempt has been made to study intense laser-induced ionic fragmentation within the framework of molecular orbital energies [10], but application of fields that last only for, say 30 as, implies an energy uncertainty of $\sim 22 \mathrm{eV}$. This makes quantal treatments of the ionization dynamics with conventional molecular states irrelevant.

Gratitude is expressed to colleagues who have contributed their mite to the acceleratorbased and intense-laser experiments on molecules in the course of several years: F. A. Rajgara, V. R. Bhardwaj, G. Ravindra Kumar, U. T. Raheja, V. Krishnamurthi, C. Badrinathan, K. Vijayalakshmi, S. Banerjee, C. P. Safvan, M. Krishnamurthy, V. Kumarappan, E. Krishnakumar, K. Nagesha, and A. K. Sinha. The high-energy femtosecond laser was partially funded by the Department of Science and Technology. Some experiments were conducted at the Nuclear Science Centre, New Delhi. 


\section{REFERENCES}

[1] J. D. Jackson, in Classical Electrodynamics (Wiley, New York, 1962), Chap. 15.

[2] S. Keller et al., Phys. Rev. A 55, 4215 (1997), R. Moshammer et al., Phys. Rev. Lett. 79, 3621 (1997).

[3] K. Boyer et al., IEEE Trans. Plasma Sci. 16, 541 (1988), T. S. Luk et al., Phys. Rev. A 48, 1359 (1993).

[4] V. R. Bhardwaj et al., Phys. Rev. A 58, 3849 (1998), ibid. 59, 3105 (1999).

[5] D. Mathur et al., Phys. Rev. A 50, R7 (1994), J. Phys. B 27, L603 (1994).

[6] T. Tonuma et al., J. Phys. B 17, L317 (1984).

[7] A. S. Schlachter et al., Phys. Scr. T3, 143 (1983).

[8] S. Banerjee et al., J. Phys. B 32, 4277 (1999), Phys. Rev. A 60, R3369 (1999).

[9] V. R. Bhardwaj et al., Phys. Rev. A 59, 1392 (1999), H. J. Neusser et al., Int. J. Mass Spectrom. Ion Processes 60, 147 (1984), and references therein.

[10] H. Rottke et al., J. Phys. B 31, 1083 (1998). 


\section{FIGURES}

FIG. 1. The radial and axial distribution of applied field within the interaction volume for $\mathrm{Si}^{8+}{ }_{-} \mathrm{C}_{6} \mathrm{H}_{6}$ collisions. The magnitude of the field is expressed in terms of an effective intensity in order to aid comparison with the equivalent distribution pattern obtained in laser- $\mathrm{C}_{6} \mathrm{H}_{6}$ interactions.

FIG. 2. The spatial distribution of applied field within the focal volume for laser- $\mathrm{C}_{6} H_{6}$ interactions (see text).

FIG. 3. Ion fragmentation patterns obtained upon irradiation of $C_{6} H_{6}$ by a) $100-\mathrm{MeV} \mathrm{Si}^{3+}$ ions (similar patterns were obtained using $\mathrm{Si}^{8+}$ and $\mathrm{F}^{7+}$ ions), and b) 35-ps-long laser pulses of $532 \mathrm{~nm}$ wavelength and peak intensity of $8 \times 10^{13} \mathrm{~W} \mathrm{~cm}^{-2}$.

FIG. 4. Fragmentation of $C_{6} H_{6}$ by a) $110-\mathrm{MeV} \mathrm{Si}^{8+}$ ions and b) $100-$ fs-long laser pulses of 806 $\mathrm{nm}$ wavelength and peak intensity of $5 \times 10^{15} \mathrm{~W} \mathrm{~cm}^{-2}$. 



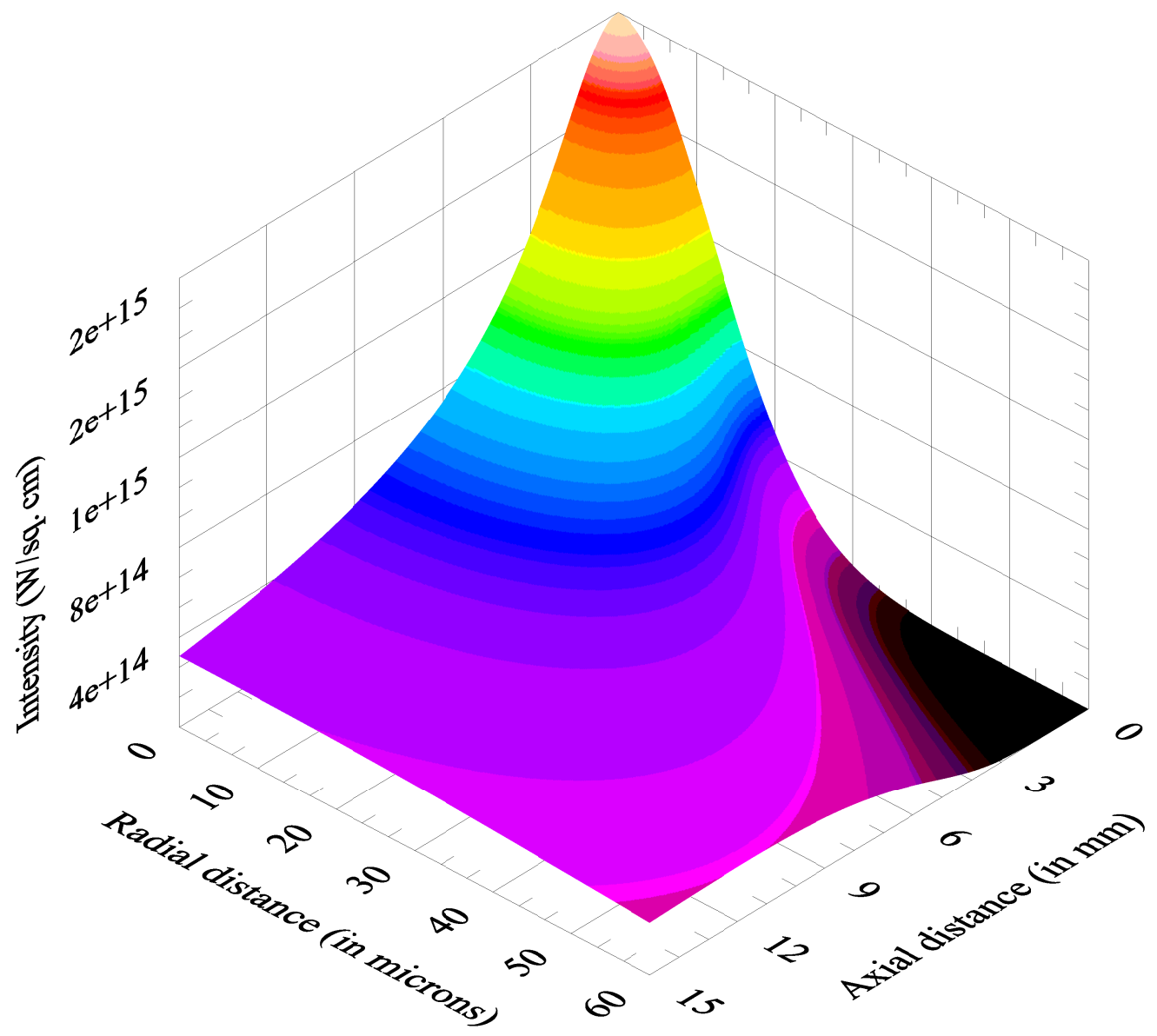




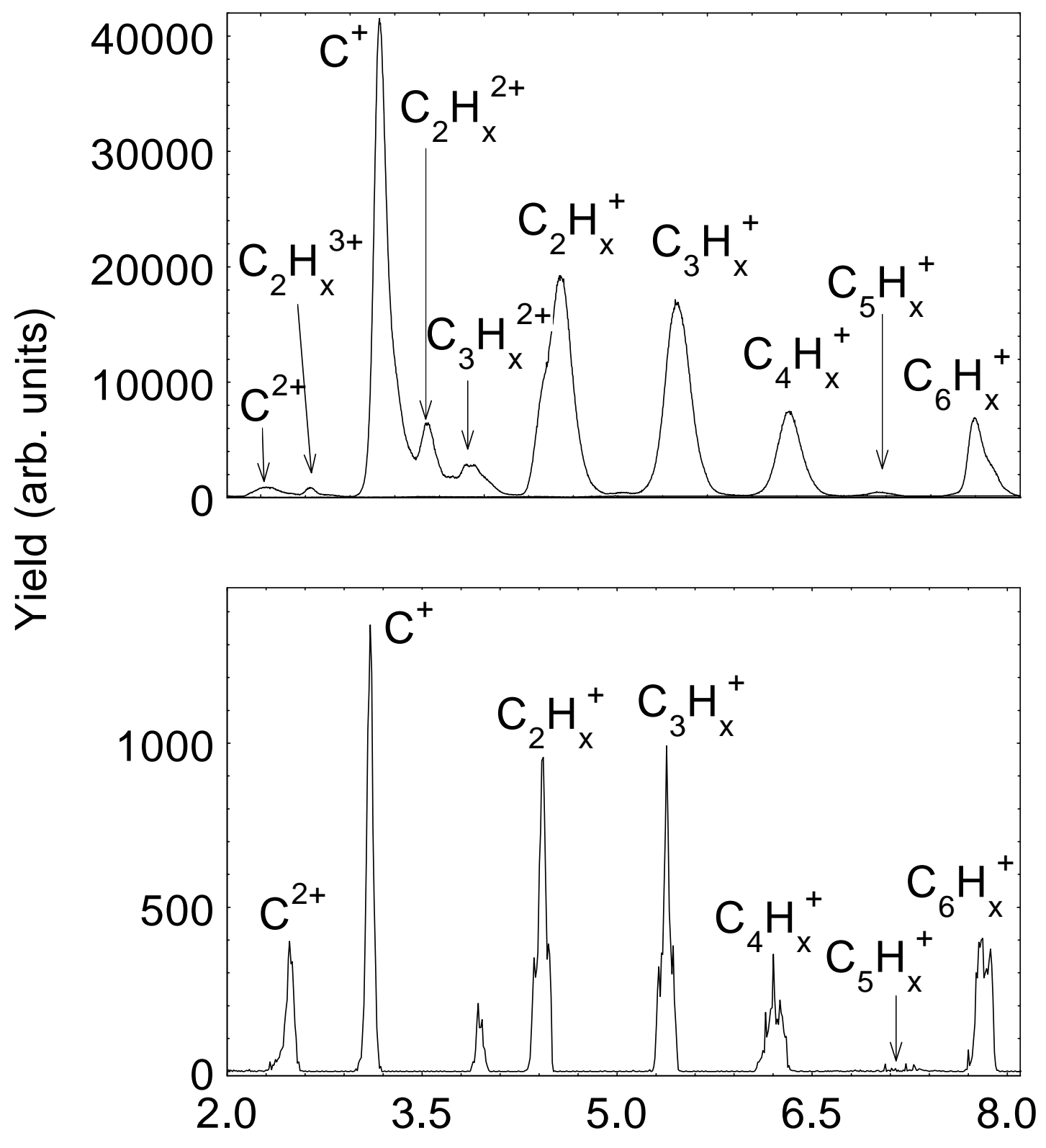

a)

b)

Flight time ( $\mu \mathrm{s})$ 


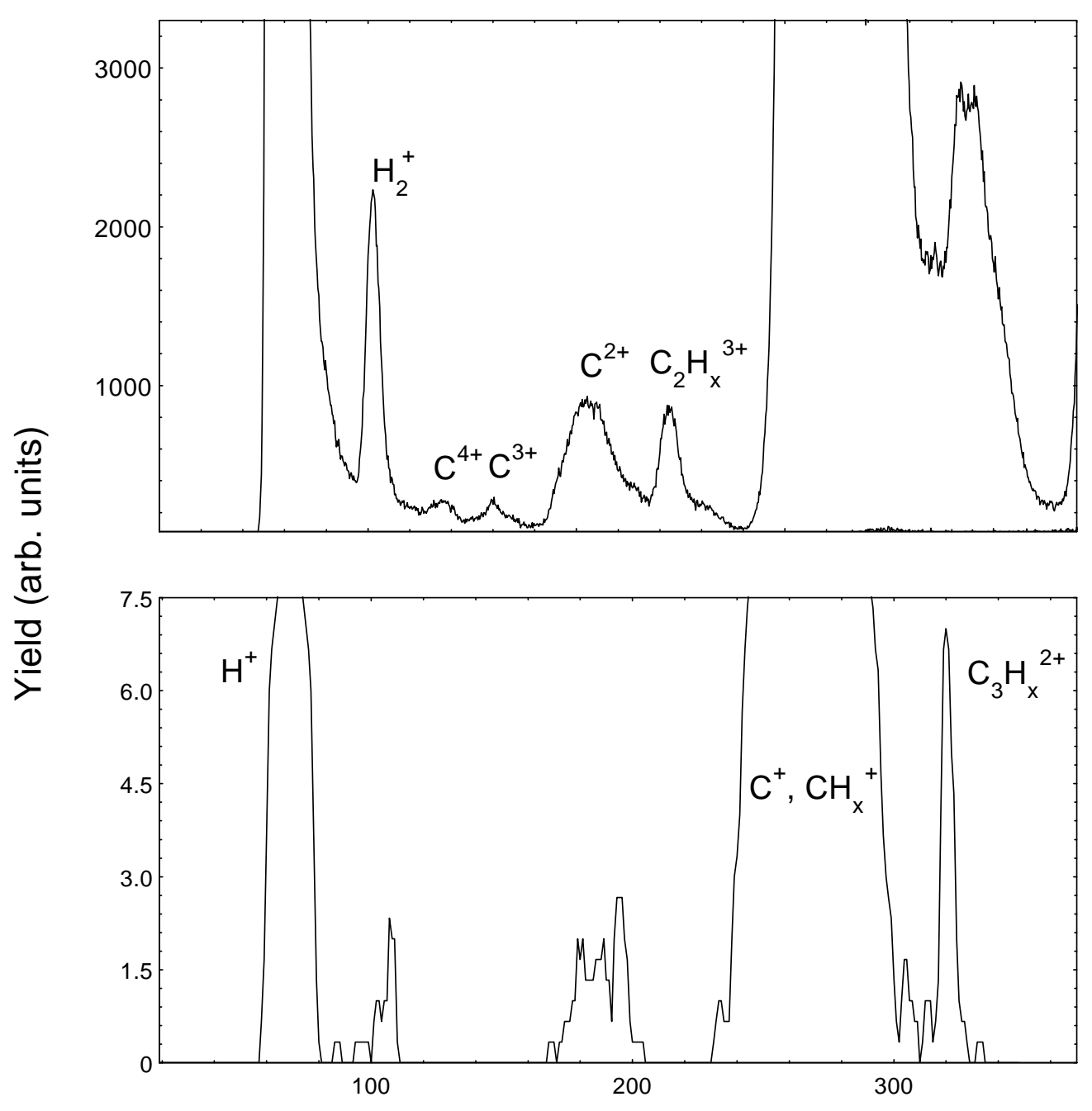

a)

b)

Flight time (ns) 